\title{
EDITORIAL
}

\section{Lung transplantation: beyond palliation}

\author{
R.D. Levy*, M. Estenne", W. Weder", M.G. Cosio
}

Some 4 decades ago, the first thoracic transplant of the modern era was performed. It was a heart, it was carried out in South Africa and although it did not last long, it opened a new door for treating patients dying of advanced cardiopulmonary conditions. Also, possibly a new door towards immortality?

Perhaps that first heart transplant was too daring for its time, as surely there were many unknowns. However, it indisputably revealed the serious potential for clinical thoracic organ transplantation. Surgeons, physicians and immunologists were stimulated to work together towards improving the understanding of problems related to organ preservation, rejection and the optimising of recipient outcomes.

The first successful lung transplants were reported $\sim 20 \mathrm{yrs}$ ago, although a number of earlier bold attempts had been made. Lungs presented a special unique problem, related to continuous unprotected exposure of the allograft to the outside environment. After all, humans breathe over 7,200 L of air daily, along with its attendant microbes, particulates and pollutants. Could a new immunosuppressed lung handle that? It seems it most certainly can.

Despite tentative early concerns, lung allografts perform remarkably well in both the short and longer term. Lung transplantation has become an important treatment option for certain carefully selected patients with a wide array of advanced cardiorespiratory diseases. Over 15,000 lung transplants have been performed in the 20 yrs since the procedure has become a clinical reality, and $\sim 1,400$ new transplants are performed annually in approximately 100 centres worldwide. Lung transplantation has become a real therapeutic option, not just palliation, for certain patients suffering from advanced emphysema, cystic fibrosis, pulmonary fibrosis and other conditions.

Since lung transplantation has become a standard and widely available option for carefully selected patients with advanced cardiopulmonary diseases, it seems timely to bring the respiratory community up to date with its current status, to review the remarkable achievements, and highlight the continuing challenges. Since pulmonologists and thoracic surgeons are the gatekeepers to the lung transplantation process, it is essential for the general respiratory community to have a good understanding of when to refer for transplantation, and when not to unreasonably raise the expectations of patients unlikely to benefit. Furthermore, with increasing numbers of lung transplant recipients receiving follow-up care in the community, it is important for respiratory specialists to understand the major issues related

\footnotetext{
*British Columbia Transplant Society, University of British Columbia, Vancouver, BC, Canada. ${ }^{\#}$ Chest Service, Erasme University Hospital, Brussels, Belgium. 'University Hospital, Thoracic Unit, Zurich, Switzerland. ${ }^{+}$Respiratory Division, Royal Victoria Hospital, McGill University, Montreal, Quebec, Canada.

Correspondence: R.D. Levy, British Columbia Transplant Society, 1081 Burrard Street, Vancouver, BC, Canada, V6Z 1Y6. Fax: 604 8068722. E-mail: rlevy@providencehealth.bc.ca
}

to the care of these patients, as well as to recognise when more specialised input from a lung transplant centre is necessary.

In order to meet these needs, the Editorial Board of the European Respiratory Journal (ERJ) has commissioned a series of state-of-the-art articles relating to pertinent topics in lung transplantation. A group of eminent physicians and scientists from around the world, leaders in the field of lung transplantation, agreed to participate. Authors were charged with not only reviewing the respective topics, but also with providing insights into the major challenges. Some of the sections will overlap somewhat with a comprehensive European Respiratory Monograph on lung transplantation also to be published in 2003. The present ERJ series has been specifically developed to provide a broad perspective, targeting topics relevant to practicing and academic respiratory specialists, and the authors hope that it will reach a wide readership.

In the article entitled "Lung transplant outcomes: a review of survival, graft function, physiology, health-related quality of life and cost-effectiveness" STUDER et al. [1] states that anticipated survival rates following lung transplantation are $\sim 75 \%$ at $1 \mathrm{yr}$ and $50 \%$ at 5 yrs. Furthermore, a number of dedicated centres have reported substantially better results with 1 -yr survival of $\sim 85 \%$. Beyond achieving a survival advantage, lung transplant recipients can also realise very substantial improvements in functional capacity and healthrelated quality of life. Lung transplantation has clearly come a long way since the early days and continues to improve.

Much of the improvement in outcomes over the past 2 decades is attributable to steady advances in areas such as donor/recipient selection, operative technique and postoperative care. The availability of newer immunosuppressive agents with greater efficacy and more favourable side-effect profiles has also had a major impact. Equally important has been the application of new concepts in management of immunosuppressive agents utilising innovations in the areas of pharmacokinetics, pharmacodynamics and therapeutic drug monitoring. In the article entitled "Immunosuppressive therapy after lung transplantation" KNOOP et al. [2] will review how this has been accomplished and how patients should now be treated.

KotlofF and Ahya [3] review the major advances that have taken place in the identification and management of "Medical complications of lung transplantation". Importantly, they specifically highlight how the development of new antiviral and antifungal agents has resulted in substantial reductions in morbidity and mortality. Furthermore, systematic utilisation of anti-infective surveillance and prophylactic strategies has resulted in substantial freedom from infection with pathogens that previously devastated solid organ transplant recipients.

However, major problems remain. Despite the successes achieved to date in clinical lung transplantation, long-term outcomes remain suboptimal. The major intermediate/longterm complication of lung transplantation affecting morbidity and mortality is bronchiolitis obliterans, a chronic progressive 
form of allograft dysfunction with airflow limitation due to small airways obstruction, thought to be a manifestation of chronic rejection. BOEHLER and ESTENNE [4] will review this very important topic in the article entitled "Post-lung transplant bronchiolitis obliterans".

Two major issues remain paramount in preventing the full potential of clinical lung transplantation being realised. The first is the paucity of donor organs. Lung transplantation volume continues to be limited by the number of available cadaveric donors, resulting in substantial mortality on transplant waiting lists. Major efforts are being expended in order to optimise and expand the limited pool of solid organ donors. A number of innovative strategies are currently evolving in order to increase the number of potential donor organs, including maximising donor lung preservation time and viability, the use of "marginal" or "suboptimal" donors, the use of living donors for lobar transplantation, as well as the use of nonheart-beating donors. This extremely important and challenging topic will be the subject of the article "Strategies to increase limited lung donor resources" by DE PERROT et al. [5].

Equally important and intimately related to the donor shortage is the proper identification of optimal patients for lung transplantation. This issue of the ERJ leads of the series on lung transplantation with an article entitled "Indications, patient selection and timing of referral for lung transplantation", by GLANVILLE and ESTENNE [6]. This important article will advise that outcomes post-transplant must be viewed in the context of an individual patient's prognosis without transplantation. Research is currently being conducted exploring large data registries in order to try to identify risk factors for poor patient outcome, both with and without lung transplantation. This type of data has the potential to become increasingly useful in identifying which patients are likely to derive the greatest benefit from lung transplantation. This information is essential in order to counsel the individual patient regarding the appropriateness and optimal timing for transplant, as well as for deriving objec-

tive strategies for allocating the limited number of organs in the most judicious fashion from a societal perspective.
Last (but certainly not least) MALLORY and SPRAY [7] will bring readers up to date with the very important area of "Paediatric lung transplantation".

To date, many respiratory specialists have relegated lung transplantation to the realm of "last ditch" efforts for the dying patient, with little recognition of the potential for lung transplantation to provide substantial benefits in terms of quality of life. This has in a sense developed into a selffulfilling prophecy for those who focus on the shortcomings of lung transplantation, with patients often being referred both too late to identify a suitable donor and so sick that outcomes are predictably suboptimal. For colleagues who continue to consider lung transplantation a palliative procedure, perhaps it's time for a fresh look. The authors hope to help focus this "fresh look" by bringing this collection of articles to the readers.

\section{References}

1. Studer SM, Orens JB, Levy RD, McNeil K. Lung transplant outcomes: a review of survival, graft function, physiology, health-related quality of life and cost-effectiveness. Eur Respir $J 2004$ (in press).

2. Knoop C, Haverich A, Fischer S. Immunosuppressive therapy after lung transplantation. Eur Respir J 2004 (in press).

3. Kotloff RM, Ahya VN. Medical complications of lung transplantation. Eur Respir J 2004 (in press).

4. Boehler A, Estenne M. Post-transplant bronchiolitis obliterans. Eur Respir J 2003 (in press).

5. de Perrot M, Weder W, Patterson GA, Keshavjee S. Strategies to increase limited lung donor resources. Eur Respir J 2004 (in press).

6. Glanville AR, Estenne M. Indications, patient selection and timing of referral for lung transplantation. Eur Respir J 2003; 22: 845-852.

7. Mallory GB, Spray TL. Paediatric lung transplantation. Eur Respir J 2004 (in press). 